\title{
Oscillations and DNA Repair in a Spatio-Temporal Model of the p53 Signalling Pathway
}

\author{
A. J. Terry* \\ Division of Mathematics, University of Dundee, Dundee, DD1 4HN, UK.
}

\begin{abstract}
In mammalian cells, the p53 pathway regulates the response to a variety of stresses, including oncogene activation, heat and cold shock, and DNA damage. Here we explore a mathematical model of this pathway, composed of a system of partial differential equations. In our model, the p53 pathway is activated by a DNA-compromising event of short duration. As is typical for mathematical models of the p53 pathway, our model contains a negative feedback loop representing interactions between the p53 and Mdm2 proteins. A novel feature of our model is that we combine a spatio-temporal approach with the appearance and repair of DNA damage.

We investigate the behaviour of our model through numerical simulations. By ignoring the possibility of DNA repair, we first explore the scenario in which the cell has a very inefficient DNA repair mechanism. We find that spatio-temporal oscillations in p53 and Mdm2 may occur, consistent with experimental data. We then allow p53 to be directly involved in repairing DNA damage, since experimental evidence suggests this can happen. We find that oscillations in p53 and Mdm2 can still occur, but their amplitude damps down quickly as the DNA damage is repaired. Finally, we find that a minor change to the location of the DNA damage can notably change the spatial distribution of p53 within the nucleus. We discuss the biological implications of our results.
\end{abstract}

Keywords and phrases: p53, Mdm2, DNA damage, DNA repair, cancer, spatio-temporal model, genetic oscillator, negative feedback

Mathematics Subject Classification: 92C37, 92C40, 92C42, 92C45

\section{Introduction}

In mammalian cells, the p53 protein is a transcription factor with hundreds of target genes [31, 43]. It plays a critical role in preserving genomic integrity [18]. It therefore helps to protect cells from turning cancerous, and indeed mutations in the p53 gene occur frequently in many common cancers [43]. The p53 protein, together with its activators and downstreams targets, comprise the p53 signalling pathway, or, more concisely, the p53 pathway [37].

In non-stressed conditions, levels of p53 are kept low by its rapid degradation, a process stimulated by the Mdm2 protein [10]. When cellular stresses cause DNA damage, this damage leads (via intermediate

*Corresponding author. E-mail: aterry.maths@outlook.com 
kinases) to the destabilisation of $\mathrm{Mdm} 2$, and the stabilisation and activation of p53. This allows the levels of active p53 to rise [31]. The rise in active p53 levels initiates a transcriptional program that can lead to DNA repair or apoptosis depending on whether the DNA damage is, respectively, minimal or extensive [10].

Intriguingly, the Mdm2 gene is one of the targets of p53, so that a negative feedback loop connects $\mathrm{p} 53$ and Mdm2 which can be summarised thus: p53 $\rightarrow$ Mdm2 - p53. Negative feedback loops are frequently associated with oscillatory dynamics [24], and indeed such dynamics have been observed for p53 and Mdm2 in cells subjected to DNA damage through exposure to irradiation or radiomimetic drugs $[13,17]$. The dynamical consequences of the p53-Mdm2 negative feedback loop have been explored in numerous mathematical models. Such models have assumed a variety of forms, including ordinary differential equations (ODEs) [10,45], delay differential equations (DDEs) [3, 19,23,24], and partial differential equations (PDEs) $[12,39,40]$. There have also been models containing a stochastic component $[28,29,31]$.

Every modelling approach has its own particular advantages and disadvantages. A significant advantage enjoyed by a PDE model is that it can explicitly represent the following fact: the rate at which a reaction will occur at a specific location inside a cell will depend on the local concentrations of the reacting species there.

In view of the discussion above, we set as our objective the exploration of a PDE model of the p53 pathway. The main novel feature of our model is that we combine a spatio-temporal approach with the appearance and repair of DNA damage. We will see that the appearance of DNA damage leads to spatio-temporal oscillations in p53 and Mdm2, and that these oscillations damp down to equilibrium behaviour as the DNA damage is repaired. We will also see how a small change to the spatial assumptions in our model can lead to clear differences in the spatio-temporal results without significantly altering the temporal results.

We outline the rest of this paper. In sections 2 and 3, we describe the kinetics of the p53 pathway and formulate a PDE model, based on these kinetics. Computational results from our model are presented in section 4. In section 5, we draw conclusions and consider ways to extend our work. Some technical details and additional results are deferred to a Supporting Information text file, and computational movie clips are included as Supporting Information files.

\section{Kinetics of the p53 pathway}

In figure 1, we show a schematic of the p53 pathway. It is on the basis of this schematic that we have formulated our mathematical model. The schematic does not depict the p53 pathway in its entirety but focuses on several particular features which we describe in this section.

Although p53 is synthesised in the cytoplasm, it must translocate to the nucleus in order to perform its transcriptional role. For simplicity, we show p53 as being present only in the nucleus in figure 1, but we will allow p53 to be present in both the cytoplasm and the nucleus in our mathematical model. Note that the translocation of p53 to the nucleus is assisted by its possession of a nuclear localisation signal [21]. Also, newly-synthesised p53 is inactive as a transcription factor and becomes activated when it is phosphorylated. We denote inactive p53 and active p53 by, respectively, p53i and p 53p in figure 1 . We will assume in our model that p53i appears at a constant rate in the cytoplasm (an analogous assumption is made in the p53 model in [39]).

Synthesis of Mdm2 proteins occurs in the cytoplasm. In order to enter the nucleus, Mdm2 must be phosphorylated by active or phosphorylated Akt $[22,31]$. The process by which Akt itself becomes activated involves interactions at the cell membrane which we shall not concern ourselves with, although details on them may be found in [31]. We shall simply assume in our model that active Akt appears at a constant rate in the vicinity of the cell membrane. Once phosphorylated, Mdm2 may travel freely from the cytoplasm to the nucleus and vice versa [31]. For simplicity, and as in the p53 model in [31], we shall, in our model, ignore the possibility that phosphorylated Mdm2 in the nucleus can become spontaneously unphosphorylated. In figure 1, we denote active (or phosphorylated) Akt, unphosphorylated Mdm2, and phosphorylated Mdm2 by, respectively, Aktp, Mdm2i and Mdm2p. 


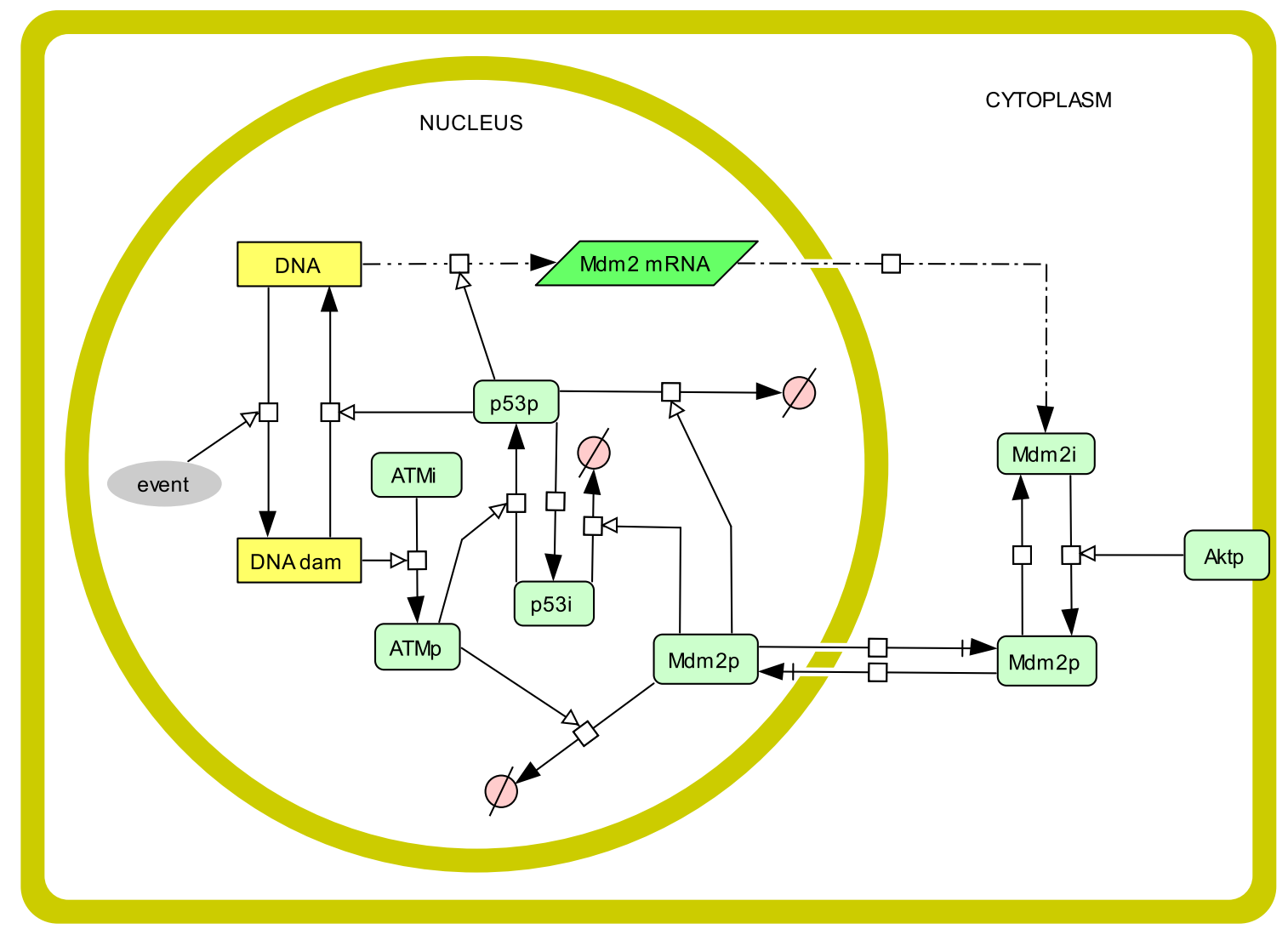

FiguRE 1. Cell circuitry schematic showing interactions in the p53 pathway. The letter "i" or "p" at the end of the name of a species denotes, respectively, an unphosphorylated (inactive) or a phosphorylated (active) form. The terms "DNA dam" and "event" are abbreviations for, respectively, "DNA damage" and "DNA-compromising event".

As mentioned in the Introduction, Mdm2 stimulates p53 degradation. This may occur in either the cytoplasm or the nucleus [44]. It has been found that active Akt, by phosphorylating Mdm2, promotes Mdm2-mediated degradation of p53 [27]. Hence we assume, in figure 1 and in our model, that Mdm2, after phosphorylation by Akt, can stimulate p53 degradation. If we wished to represent Mdm2-mediated degradation of p53 in greater detail, we could do this by bearing in mind the results in [5], which suggest that the Mdm2 protein has numerous sites for its phosphorylation, and the phosphorylation of at least some of these sites appears to be necessary for Mdm2-mediated degradation of p53. We could also bear in mind that Mdm2 inhibits p53 transcriptional activity merely by binding to it [20].

Activation of the p53 pathway may occur through a number of mechanisms, including oncogene activation, heat and cold shock, and DNA damage [6,31]. The most studied of these mechanisms, both experimentally and computationally, is DNA damage [31]. DNA damage occurs routinely in the life of a cell. It may be caused, for example, by metabolic, ribosomal, or oxidative stress, or exposure to ultraviolet radiation [18]. DNA damage may be induced experimentally by subjecting a cell to gamma irradiation or radiomimetic drugs $[13,17,18]$. In figure 1 and in our model, we suppose that a generic "DNA-compromising" event causes DNA damage. The DNA-compromising event and DNA damage are labelled, respectively, as "DNA dam" and "event" in figure 1. 
Several kinases are known to respond quickly to DNA damage. In particular, ATM is rapidly activated (by phosphorylation) in response to double strand breaks (DSBs) in DNA [2]. Activated ATM then activates p53 (by phosphorylation), either directly or via the intermediate kinase Chk2, and simultaneously enhances degradation of the p53-inhibitor Mdm2 [6,18,38]. For simplicity, we suppose that direct activation by active ATM is the only form of p53 activation in figure 1 and in our model. We also suppose, consistent with experimental results [6] and a previous mathematical model [31], that Mdm2-mediated degradation occurs more slowly for active (phosphorylated) p53 than for inactive p53. In figure 1, we denote inactive ATM and active (phosphorylated) ATM by, respectively, ATMi and ATMp.

The activation of p53, together with the enhanced degradation of its inhibitor Mdm2, enables p53 to initiate a transcriptional response to DNA damage. Activated p53 upregulates production in the nucleus of mRNA transcript molecules from its target genes, which include the Mdm2 gene [36]. In figure 1, we denote Mdm2 transcript molecules by Mdm2 mRNA. Newly synthesised Mdm2 transcript molecules move out into the cytoplasm and there produce, by translation, Mdm2 proteins. The behaviour of Mdm2 proteins has been described above.

Activated p53 initiates transcription from genes whose protein products are involved in DNA repair [35]. However, note that active p53 may also be directly involved in DNA repair [26,35]. For simplicity, then, we suppose in figure 1 and in our model, that DNA damage is directly reversed by active p53. Thus, we ignore any intermediary species in this process in figure 1 and in our model.

Finally, as alluded to in the Introduction, a normal cell will undergo apoptosis if DNA damage is not repaired quickly [10,31]. This can occur, for instance, through p53-inducible pro-apoptotic genes such as PUMA and Bax [30]. On the other hand, not every cell is normal or healthy, and in particular cancer cells have impaired apoptotic mechanisms. We do not consider any mechanism for apoptosis in figure 1 or in our model, and therefore our model is most appropriate as a representation of either normal cells with minor DNA damage, or abnormal cells lacking a correct apoptotic response.

\section{The model}

As mentioned at the start of the previous section, we have used the kinetics discussed in that section and depicted in figure 1 as the basis for a mathematical model. Our model is stated fully in the Supporting Information text file that accompanies this paper. We summarise it here.

The model has three compartments - nucleus, cytoplasm, and cell membrane. The cell membrane compartment is intended to represent the region in the cell where reactions at the cell membrane occur and does not explicitly represent the cell membrane. For each species in those compartments in which it may be found, its spatio-temporal evolution is governed by a partial differential equation with two independent spatial variables, $x$ and $y$, and the independent time variable $t$. Thus, our model has two spatial dimensions and gives a cross section of dynamics inside a cell. The nucleus is assumed to be a circle, the cytoplasm is an annular region surrounding the nucleus, and the cell membrane is a very thin annulus surrounding the cytoplasm.

We make spatial assumptions regarding the processes of translation and transcription. Translation is assumed to occur in an annular region throughout the middle of the cytoplasm, and not right next to the nuclear membrane or cell membrane. Similar assumptions regarding translation are made in the models in [39-42]. We assume that transcription occurs in a circular region in the centre of the nucleus. This is more realistic than the assumption, made in related spatio-temporal modelling studies [39-42], that transcription occurs throughout the entire space of the nucleus. In living cells, transcription occurs at genes in the nucleus, where there are typically two gene copies per gene type [32], although there can be more [34], and in particular gene copy number for some gene types is known to be elevated (or "amplified") in certain forms of cancer [9].

The general form for each equation is a reaction-diffusion-convection equation. Thus, if we denote the concentration of a species $w$ at the point $(x, y)$ at time $t$ by $[w(x, y, t)]=[w]$, then the equation for $w$, in 
a compartment in which it may be found, takes the form

$$
\frac{\partial[w]}{\partial t}=D_{w} \nabla^{2}[w]+R-\theta_{r} \nabla \cdot\left([w] \mathbf{a}_{w}\right) .
$$

Here $D_{w}$ is the diffusion rate for $w$. This is assumed to be a positive constant for each species except the variable representing DNA damage, for which the diffusion rate is taken to be a very small positive constant whilst the DNA-compromising event occurs and is otherwise zero. The term $R$ represents a reaction, such as translation or transcription. The final term in (3.1), namely $-\theta_{r} \nabla \cdot\left([w] \mathbf{a}_{w}\right)$, is a convection term that represents cytoplasmic active transport, that is, facilitated transport along cytoskeletal elements called microtubules. In this convection term, $\mathbf{a}_{w}$ is a vector, with constant magnitude or speed $a_{w}$, that is directed towards the centre of the nucleus. Active transport is assumed to occur for only three species in the cytoplasm; in all other cases, the speed of the convection of a species in a compartment is assumed to be zero. The three species actively transported in the cytoplasm are (using the notation from section 2) p53i, p53p, and ATMi. Our assumption of active transport for p53i and p53p is consistent with a similar assumption in the p53 model in [40] and with experimental results on p53 association with microtubules [14]. Also, our assumption of active transport for ATMi towards the nucleus is made on account of the critical role of ATMi in sensing DNA damage in the nucleus and since several studies have found ATM to be present predominantly within the nucleus [16]. Finally, in the convection term, $\theta_{r}$ is a spatial function which is equal to 0 at those points in the cytoplasm in close proximity to the cell or nuclear membranes and otherwise equals 1 . This spatial function is included because microtubules do not necessarily touch these membranes [40].

We illustrate our model by presenting one of the equations in it, namely the equation for Mdm2 transcript in the nucleus:

$$
\frac{\partial\left[M d m 2_{t}\right]}{\partial t}=D_{M d m 2 t} \nabla^{2}\left[M d m 2_{t}\right]+\theta_{r_{1}} B_{1}+\theta_{r_{1}} B_{2}\left(\frac{\left[p 53_{p}\right]^{h_{p}}}{(K)^{h_{p}}+\left[p 53_{p}\right]^{h_{p}}}\right)-d_{M d m 2 t}\left[M d m 2_{t}\right] .
$$

Here $\left[M d m 2_{t}\right]$ and $\left[p 53_{p}\right]$ are the concentrations of Mdm2 transcript and phosphorylated p53, respectively. The terms on the right hand side of (3.2) represent, in the following order, diffusion, p53-independent production of Mdm2 transcript, p53-dependent production of Mdm2 transcript, and spontaneous (or constitutive) degradation. Note that $D_{M d m 2 t}, B_{1}, B_{2}, h_{p}, K, d_{M d m 2 t}$ are all positive constants, whilst $\theta_{r_{1}}$ is a spatial function that restricts mRNA production to a region in the centre of the nucleus.

As in previous spatio-temporal models of the p53 pathway in $[39,40]$, we choose zero initial conditions for all species in all cellular compartments. For boundary conditions, we choose conditions such that no molecules enter regions where they are not permitted to enter, that no molecules leave the cell, and that no molecules crossing a boundary are lost whilst doing so. There are three boundaries in our model the outer boundary of the cell membrane region (which is also the outside of the cell in our model), the inner boundary of the cell membrane region, and the nuclear membrane. Only Aktp moves across the inner cell membrane boundary, and to represent this we choose continuity of flux boundary conditions. For those species moving across the nuclear membrane, we choose, as in the spatio-temporal model of the p53 pathway in [40], continuity of flux boundary conditions with a thin boundary layer, where diffusion through this layer is slower than in the cytoplasm or nucleus to represent the fact that transport through the nuclear membrane is restricted to narrow channels called nuclear pore complexes [7]. Otherwise we choose zero flux boundary conditions. Initial and boundary conditions are stated explicitly in the Supporting Information text file. The system is simulated until it approaches an equilibrium, and then a DNA-compromising event is used to activate the p53 pathway by causing DNA damage in a circular region in the nucleus. We present our results in the next section.

\section{Results}

To solve our model, we used the same method of solution as in recent related spatio-temporal modelling studies of intracellular signalling pathways [39-42]. This method can be summarised as follows. First we 
non-dimensionalised our model by scaling all of the variables by reference values. Second, we found values for the non-dimensionalised parameters by performing a simulation study of the non-dimensionalised model in which we sought certain qualitative behaviours, described in the next paragraph. In our simulation study, we numerically solved the non-dimensionalised model using the finite element method, as implemented by the software package COMSOL. We used version 3.5 of this package. Third, we estimated the reference values and, using these and the non-dimensional parameter values, we calculated the dimensional parameter values.

What qualitative behaviours did we seek in our simulation study of the non-dimensionalised model? We began by seeking (and finding), in the submodel that ignores DNA repair, oscillatory dynamics in p53 and Mdm2 after the DNA-compromising event. We expected to be able to find such dynamics because, in this submodel, the p53 pathway is continuously stimulated after the DNA-compromising event by unrepaired DNA damage, and p53 and Mdm2 are linked by a negative feedback loop. Moreover, such dynamics are found in other modelling studies $[10,24,31,39,40]$ and in experiments with PTEN knockouted cells which proliferated after irradiation, suggesting they had inefficient cell cycle arrest and DNA repair mechanisms $[13,17,31]$. Having found oscillatory dynamics in the submodel that ignores DNA repair, only three parameters were left undetermined, namely those governing DNA repair by p53. We completed our simulation study by investigating the model behaviour for different values of these three parameters, seeking (and finding) DNA repair within a reasonably short time after the DNA-compromising event. We interpreted a "reasonably short time" to mean "not more than three or four times the oscillatory period of p53". We sought reasonably fast DNA repair because, as noted in the final paragraph of section 2, our model is suitable as a representation of a normal or healthy cell with minor DNA damage, and we might expect minor DNA damage to be repaired quickly. To faciliate our simulation study, we began by choosing non-dimensional parameter values to be of similar magnitude to analogous non-dimensional parameter values in [39-42], where analogous parameters existed.

In the simulation study described in the previous paragraph, all of the spatial assumptions in our model satisfied radial symmetry about the cell centre. Thus, for example, the following three points were all assumed to coincide: the centre of the region of DNA damage, the centre of the nucleus, and the cell centre. To explore the spatial aspect of our model in more detail, we considered additional cases in which the radial symmetry was broken. Specifically, we shifted the centre of the region of DNA damage to the left of the centre of the nucleus by a distance of one-tenth of the nuclear diameter. Although this was only a minor change to the spatial assumptions, the impact on the spatio-temporal behaviour of the model was quite noticeable. We have collected our results into four cases, which are summarised in table 1 and discussed in subsections 4.1 to 4.4 below.

Full details on our method of solution, together with all the parameter values, may be found in the Supporting Information text file. We comment on some of the dimensional parameter values here. Our value for protein diffusion rates in the cytoplasm and nucleus is $0.0047 \mu \mathrm{m}^{2} \mathrm{~s}^{-1}$, and our value for mRNA transcript diffusion rates in the cytoplasm and nucleus is $0.0095 \mu \mathrm{m}^{2} \mathrm{~s}^{-1}$. We deliberately assumed that mRNA transcript molecules diffuse faster than protein molecules, since mRNA transcript molecules are smaller than protein molecules. Our diffusion rates are of the same order of magnitude as diffusion rates in the modelling studies in [39-42]. Our value for the cytoplasmic active transport rates, for p53i, p53p, and ATMi, is $0.0039 \mu \mathrm{ms}^{-1}$, which is consistent with analogous parameters in the spatio-temporal models of the Hes1 and p53 pathways in [40]. Note that diffusion and active transport rates are unique to spatiotemporal modelling studies such as this one and are not a feature of temporal modelling studies. Finally, for reaction rates involving Hill functions, we have used Hill coefficients equal to 1 or 2, except in the case of p53-dependent production of Mdm2 transcript for which we used a Hill coefficient of 4 . These values for Hill coefficients are largely consistent with previous modelling studies $[10,31,32,39,40]$

\subsection{Case 1: Oscillations}

In figures 2 and 3, we present results for our model for the case (case 1) in which: (i) the centre of the region of DNA damage coincides with the centre of the nucleus; (ii) DNA repair by p53 is ignored. In 


\begin{tabular}{|c|c|c|c|c|}
\hline Case & $\begin{array}{l}\text { Centre of region of } \\
\text { DNA damage }\end{array}$ & DNA repair & $\begin{array}{l}\text { Model behaviour after } \\
\text { DNA-compromising event }\end{array}$ & Refer to \\
\hline 1 & Centre of nucleus & omitted & $\begin{array}{l}\text { sustained oscillations in p53 and } \mathrm{Mdm} 2 \\
\text { radially symmetric about the cell centre }\end{array}$ & subsection 4.1 \\
\hline 2 & Centre of nucleus & occurs & $\begin{array}{l}\text { DNA repair, with damping oscillations in } \\
\text { p53 and Mdm2; } \\
\text { radially symmetric about the cell centre }\end{array}$ & subsection 4.2 \\
\hline 3 & $\begin{array}{l}\text { Left of nuclear centre } \\
\text { by one-tenth of the } \\
\text { nuclear diameter }\end{array}$ & omitted & $\begin{array}{l}\text { sustained oscillations in p53 and } \mathrm{Mdm} 2 \text {; } \\
\text { spatially asymmetric }\end{array}$ & subsection 4.3 \\
\hline 4 & $\begin{array}{l}\text { Left of nuclear centre } \\
\text { by one-tenth of the } \\
\text { nuclear diameter }\end{array}$ & occurs & $\begin{array}{l}\text { DNA repair, with damping oscillations in } \\
\text { p53 and Mdm2; } \\
\text { spatially asymmetric }\end{array}$ & subsection 4.3 \\
\hline
\end{tabular}

TABLE 1. Summary of our results. To assist in interpretation of these results, note that the centre of the nucleus coincides with the cell centre.

view of comments above (second paragraph, section 4), we would expect to be able to find oscillatory dynamics in p53 and Mdm2 for this case, and indeed we have found this. Three computational movie clips for case 1 are included as Supporting Information files (see section 6).

Figure 2 shows plots of total concentration over time for all the species in the model. From these plots, we note the following. The species Aktp remains at steady state levels during and after the DNAcompromising event - this is a consequence of the assumption in our model that Aktp production and degradation are independent of this event and of the DNA damage caused by it. The DNA-compromising event causes the sudden appearance of DNA damage N. The DNA damage triggers activation of ATM, that is, a substantial proportion of ATMi, particularly in the nucleus, is converted to ATMp. Active ATM (ATMp) activates p53, that is, a substantial proportion of $\mathrm{p} 53 \mathrm{i}$ is converted to p53p, causing levels of p53p to rise. Active ATM also enhances degradation of Mdm2i and Mdm2p, which causes levels of Mdm2i and Mdm2p to fall. The fall in levels of Mdm2p reduces Mdm2p-mediated degradation of p53p, further allowing levels of $\mathrm{p} 53 \mathrm{p}$ to rise. The sharp rise in levels of $\mathrm{p} 53 \mathrm{p}$ results in greatly increased production of Mdm2 mRNA transcript (shown as Mdm2t), which results in greatly increased production of Mdm2i, with a proportion of the newly synthesised Mdm2i being converted to Mdm2p by Aktp in the cytoplasm. Thus, levels of Mdm2i and Mdm2p rise. The degradation of p53i and p53p is enhanced by the increase in Mdm2p levels, so that p53i and p53p levels then fall. The fall in p53p levels causes a reduction in p53p-inducible production of Mdm2 transcript, which leads to a fall in Mdm2p levels. This results in a reduction in Mdm2p-mediated degradation of p53i and p53p. Then, since we have assumed in our model that $\mathrm{p} 53 \mathrm{i}$ is produced at a constant rate, levels of $\mathrm{p} 53 \mathrm{i}$ and $\mathrm{p} 53 \mathrm{p}$ rise again. Thus, we return to the situation where p53p levels are high, and an oscillatory cycle in p53 and Mdm2 dynamics now repeats.

Figure 3 shows, over a typical period (namely, the period between the second and third troughs in p53p levels in figure 2), spatial profiles of p53i, p53p, Mdm2 mRNA transcript, Mdm2i, and Mdm2p. We make numerous observations from these spatial profiles. First, note that p53i local concentrations are highest in the cytoplasm, despite the fact that p53i is actively transported from the cytoplasm to the nucleus. We account for this by noting that $\mathrm{p} 53 \mathrm{i}$ is produced in the cytoplasm and, upon entering the nucleus, is converted to p53p by ATMp in the presence of DNA damage. (Spatial profiles of ATMi, ATMp, and DNA damage N are included in the Supporting Information text file.) Second, note that the local concentration of p53p is highest in and around the nucleus, a result consistent with experimental studies $[13,17,18]$ and a previous spatio-temporal modelling study [40]. Third, the local concentration of Mdm2 transcript is highest when the local concentration of nuclear p53p is highest, which is the behaviour we would expect given that Mdm2 transcript production is upregulated by nuclear p53p in our model (as we can see in equation (3.2)). Fourth, the high levels of nuclear Mdm2 transcript at time 848 minutes cause high levels of Mdm2i at time 914 minutes, since Mdm2i is created from Mdm2 transcript in the 

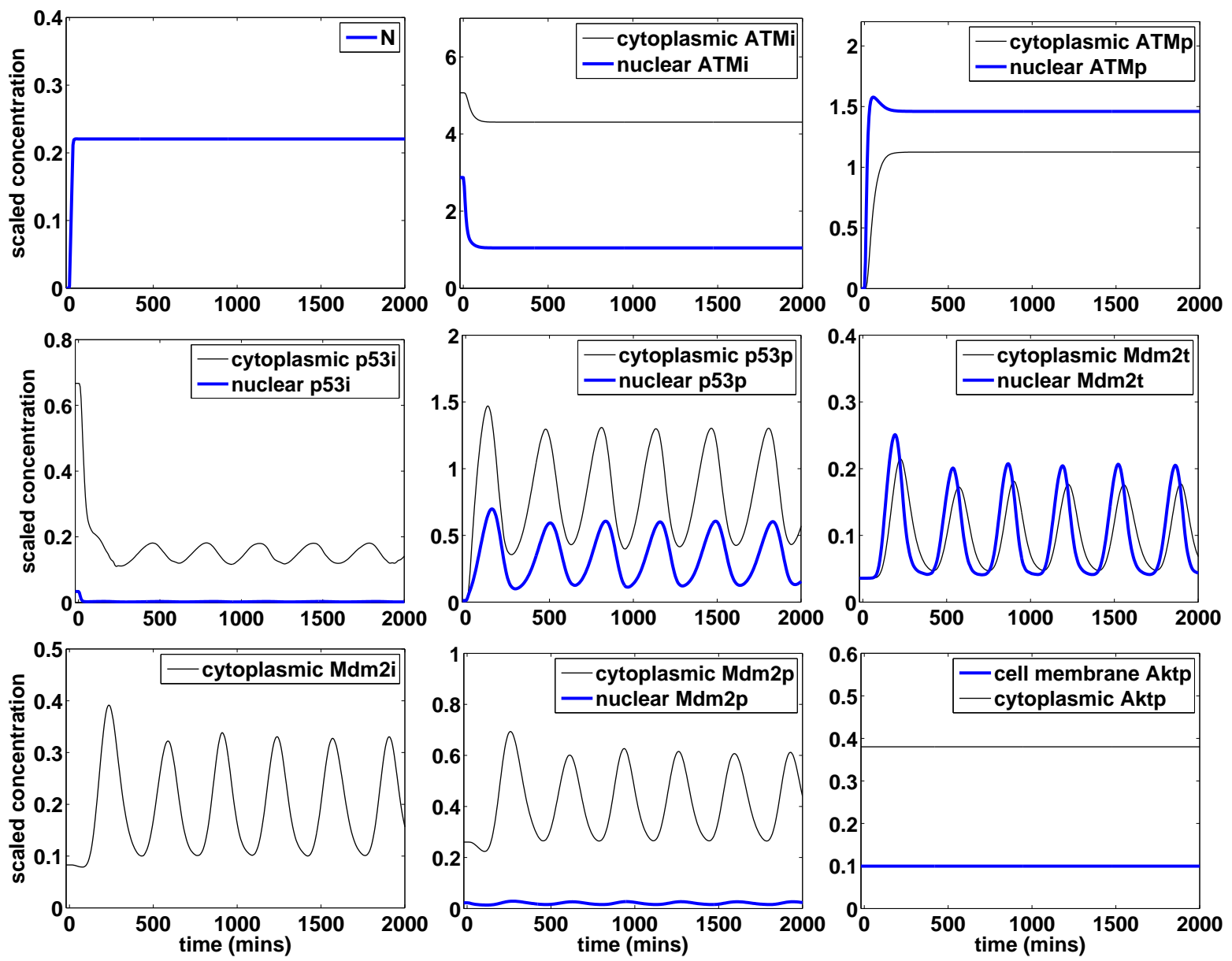

Figure 2. Plots showing total concentration over time for all species in case 1 (see subsection 4.1 for a description). Here "Mdm2t" refers to the species Mdm2 transcript. Concentrations are shown in non-dimensionalised units. Time is shown in dimensional units, with the dimensional time $t=0$ shifted to correspond to the start of the DNAcompromising event. The DNA-compromising event ends at time 21 minutes.

cytoplasm and it takes time for newly-synthesised Mdm2 transcript to diffuse outwards from the nucleus to the cytoplasm. Mdm2i is not present in the nucleus, in view of our modelling assumption, based on experiments, that it cannot be found there. Fifth, the high levels of Mdm2i at time 914 minutes cause high levels of Mdm2p at times 914 and 980 minutes, since new Mdm2p appears when newly-synthesised Mdm2i is converted to Mdm2p by Aktp. In our model, we have (as mentioned in section 2) assumed that Aktp, or active Akt, appears at a constant rate in the vicinity of the cell membrane, so the conversion of Mdm2i to Mdm2p occurs most rapidly next to the cell membrane. (Spatial profiles of Aktp are included in the Supporting Information text file.) Finally, we see that low local concentrations in p53i and p53p coincide with high local concentrations in Mdm2p, since Mdm2p enhances degradation of p53i and p53p.

\subsection{Case 2: DNA repair}

In figures 4 to 6 , we present results for our model for the case (case 2) in which: (i) the centre of the region of DNA damage coincides with the centre of the nucleus; (ii) DNA repair occurs. As explained 


$\mathrm{t}=650 \quad \mathrm{t}=716 \quad \mathrm{t}=782 \quad \mathrm{t}=848 \quad \mathrm{t}=914 \quad \mathrm{t}=980$
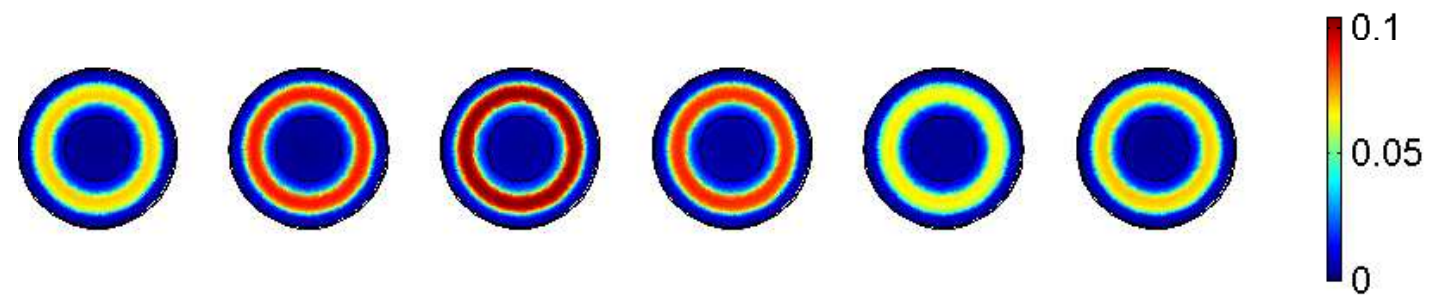

(a) p53i
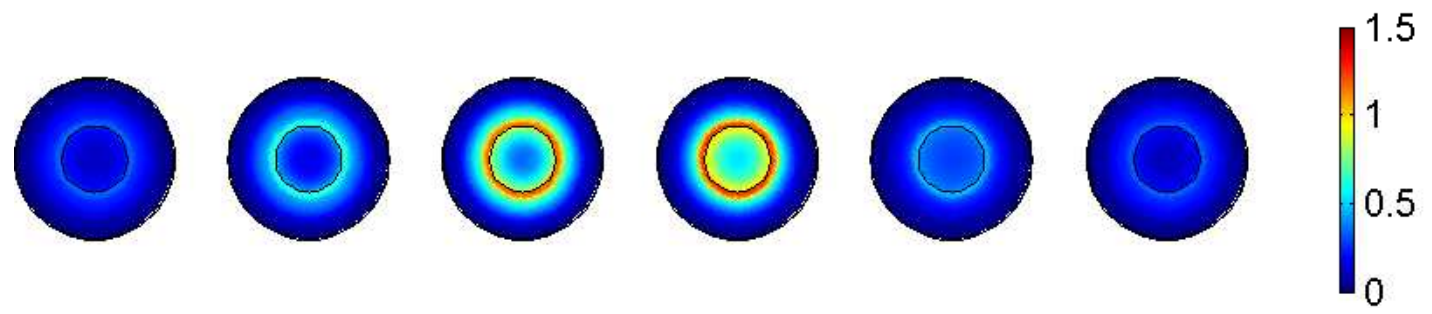

(b) p53p
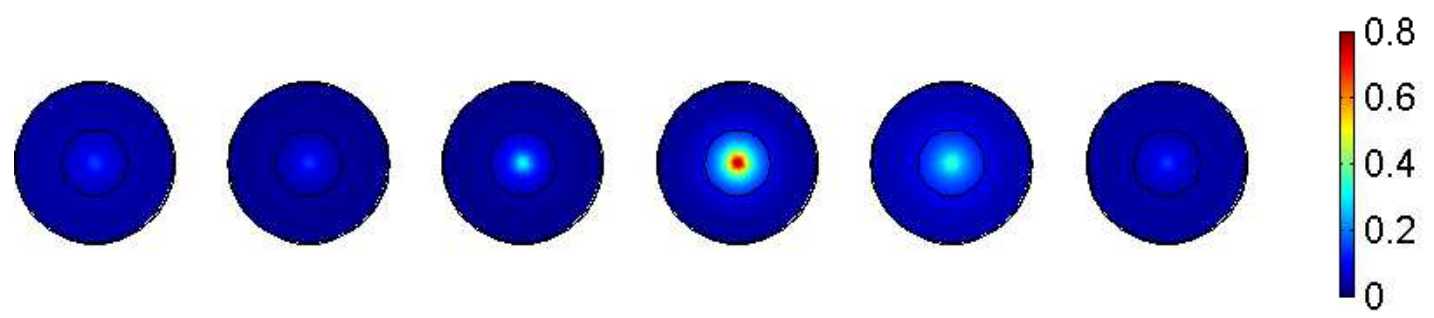

(c) Mdm2 transcript
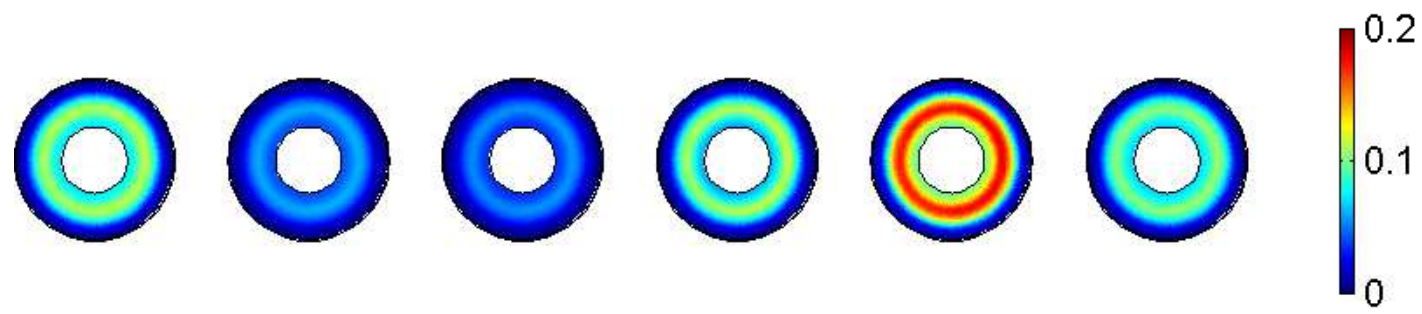

(d) Mdm2i
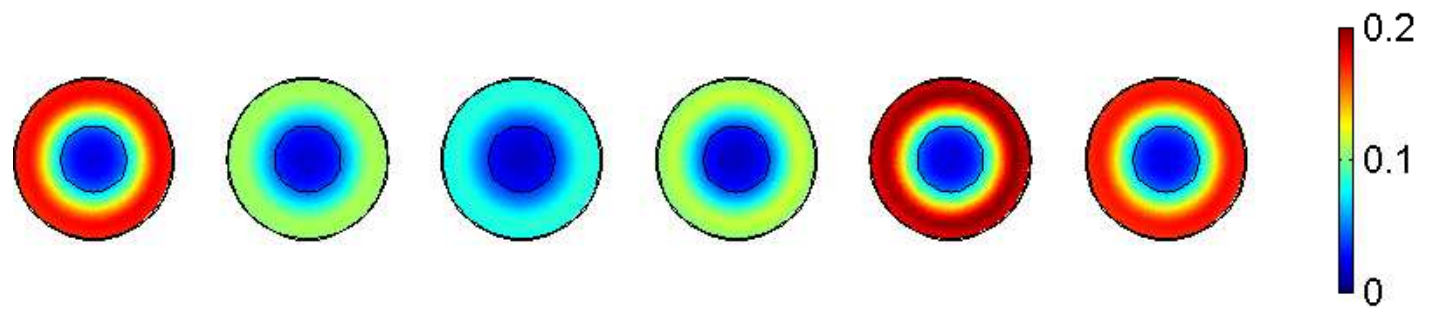

(e) $\operatorname{Mdm} 2 \mathrm{p}$

FiguRE 3. Spatial profiles of various species in case 1 (see subsection 4.1 for a description). The local concentrations are shown in non-dimensional units, and the time is in dimensional units and shifted as in figure 2. 

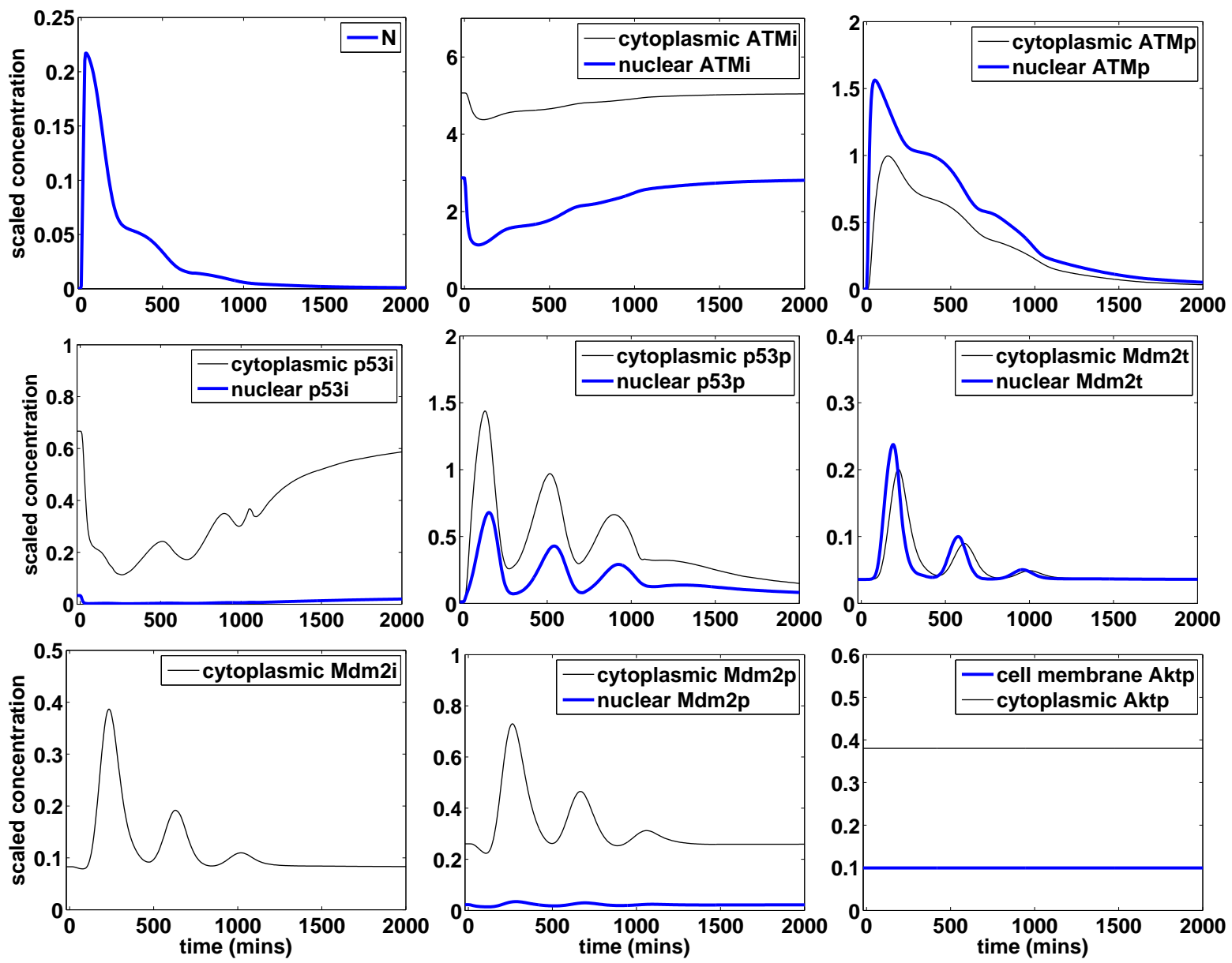

Figure 4. Plots showing total concentration over time for all species in case 2 (see subsection 4.2 for a description). Here "Mdm2t" refers to the species Mdm2 transcript. Concentrations are shown in non-dimensionalised units. Time is shown in dimensional units, with the dimensional time $t=0$ shifted to correspond to the start of the DNAcompromising event. The DNA-compromising event ends at time 21 minutes.

above (second paragraph, section 4), the qualitative behaviour that we sought was DNA repair within a reasonably short time after the DNA-compromising event, and this is indeed what we have found. Four computational movie clips for case 2 are included as Supporting Information files (see section 6).

Figure 4 shows plots of total concentration over time for all the species in the model. What do we notice from these plots? As for case 1 in figure 2, the species Aktp is unaffected by the DNAcompromising event and remains at steady state levels during and after it. Also as in figure 2: the DNA-compromising event causes the sudden appearance of DNA damage N, which triggers activation of ATMp from ATMi, especially in the nucleus; ATMp mediates activation of p53p from p53i, both directly and by upregulating degradation of the p53-inhibitor Mdm2p; and p53p creates Mdm2 transcript, thereby initiating p53-Mdm2 negative feedback interactions that lead to oscillatory dynamics. But now, unlike in case 1, figure 4 shows that p53p repairs DNA damage $\mathrm{N}$, with a notable drop in the level of DNA damage coinciding with each peak in levels of nuclear p53p. The repair of DNA damage causes a reduction in the rate of activation of ATMp, and consequently a reduction in the rate of ATMp-mediated activation 
of $\mathrm{p} 53 \mathrm{p}$. The falling rate of $\mathrm{p} 53 \mathrm{p}$ activation disrupts the $\mathrm{p} 53-\mathrm{Mdm} 2$ negative feedback balance required for stable oscillations, and so the amplitude of the oscillations diminishes, causing oscillations to cease altogether after three distinct peaks in the levels of nuclear p53p. As the oscillations damp, their period lengthens. By time 1500 minutes, the DNA damage has been almost completely repaired but not every species has returned to its equilibrium dynamics prior to the DNA-compromising event. In particular, levels of ATMp are still clearly positive, which we explain by observing that ATM activation is very sensitive to DNA damage in our model, even to very low levels of DNA damage. This property of our model is consistent with experimental evidence showing that ATM is rapidly activated in response to double strand breaks (DSBs) in DNA [2]. Given that the levels of ATMp are still positive at time 1500 minutes, the process of ATMp-mediated activation of p53 is still ongoing at this time, ensuring that levels of nuclear p53p are still positive. However, nuclear p53p levels have fallen markedly below the activation threshold for $\mathrm{p} 53 \mathrm{p}$-mediated production of Mdm2 transcript (the parameter $K$ in equation (3.2)), with the result that levels of Mdm2 transcript, Mdm2i, and Mdm2p have returned, by time 1500 minutes, to their equilibrium values prior to the DNA-compromising event.

Figures 5 and 6 show, at various times, spatial profiles for all the species in the model. What do we observe from these spatial profiles? At time 20 minutes, DNA damage $\mathrm{N}$ is clearly visible in the centre of the nucleus. This has been caused directly by the DNA-compromising event, which begins at time 0 minutes and ends at time 21 minutes. Also at time 20 minutes, we can see that ATMp is present where the DNA damage is located, but ATMi is not. We account for this by recalling that ATMi is activated (converted to ATMp) where it encounters DNA damage. By time 120 minutes, ATMp has diffused outwards into the cytoplasm. Levels of Mdm2p have fallen due to ATMp-mediated degradation. Moreover, ATMp-mediated conversion of p53i to p53p has caused levels of p53i to fall. The conversion of $\mathrm{p} 53 \mathrm{i}$ to $\mathrm{p} 53 \mathrm{p}$, together with the active transport of both $\mathrm{p} 53 \mathrm{i}$ and $\mathrm{p} 53 \mathrm{p}$ towards the nucleus, has caused p53p to appear in obvious quantities in the nucleus. By decreasing Mdm2-mediated degradation of $\mathrm{p} 53 \mathrm{i}$ and $\mathrm{p} 53 \mathrm{p}$, the reduction in levels of $\mathrm{Mdm} 2 \mathrm{p}$ may also have facilitated the appearance of $\mathrm{p} 53 \mathrm{p}$ in the nucleus. The high levels of nuclear p53p at time 120 minutes have caused a reduction in the levels of DNA damage, since p53p repairs DNA damage in our model. The high levels of nuclear p53p have additionally caused the Mdm2 gene to be "switched on", that is, Mdm2 transcript production in the centre of the nucleus has dramatically risen. This initiates the p53-Mdm2 negative feedback interactions that lead to oscillatory dynamics (observations in the final paragraph of subsection 4.1 are appropriate in the context of these dynamics). As explained in the previous paragraph, the ongoing repair of DNA damage by p53p causes a reduction in ATMi conversion to ATMp, which causes the oscillations in p53 and Mdm2 to damp. Hence, whilst there is clear evidence of the second peak in levels of nuclear p53p at time 520 minutes (figure 5(e)), the local concentrations are lower than at 120 minutes. Furthermore, by time 520 minutes, the local levels of DNA damage have fallen significantly, with corresponding behaviour in levels of ATMp. Spatial profiles for times beyond 520 minutes, for all the species in the model, are provided in the Supporting Information text file.

\subsection{Cases 3 and 4: asymmetrical spatial behaviour}

Our modelling assumptions for cases 3 and 4 are the same as for, respectively, cases 1 and 2, with one exception: the centre of the region of DNA damage is located at the centre of the nucleus in cases 1 and 2, but is located to the left of the centre of the nucleus by a distance of one-tenth of the diameter of the nucleus in cases 3 and 4 . How does this simple and seemingly minor difference in the location of DNA damage affect the model behaviour? Our temporal plot (total concentration versus time) for each species, in cases 3 and 4 , is very similar, both qualitatively and quantitatively, to the corresponding plot in cases 1 and 2, respectively (see the Supporting Information text file). However, our spatio-temporal results for cases 3 and 4 display some notable differences to the corresponding results for cases 1 and 2, respectively (see figure 7(b), the Supporting Information text file, and the computational movie clips mentioned in section 6). More precisely, our spatio-temporal results for cases 1 and 2 are radially symmetric about the cell centre (which reflects the fact that the modelling assumptions for cases 1 


$\mathrm{t}=\mathbf{2 0} \mathrm{t}=\mathbf{1 2 0} \mathrm{t}=\mathbf{2 2 0} \mathrm{t}=\mathbf{3 2 0} \quad \mathrm{t}=\mathbf{4 2 0} \quad \mathrm{t}=\mathbf{5 2 0}$
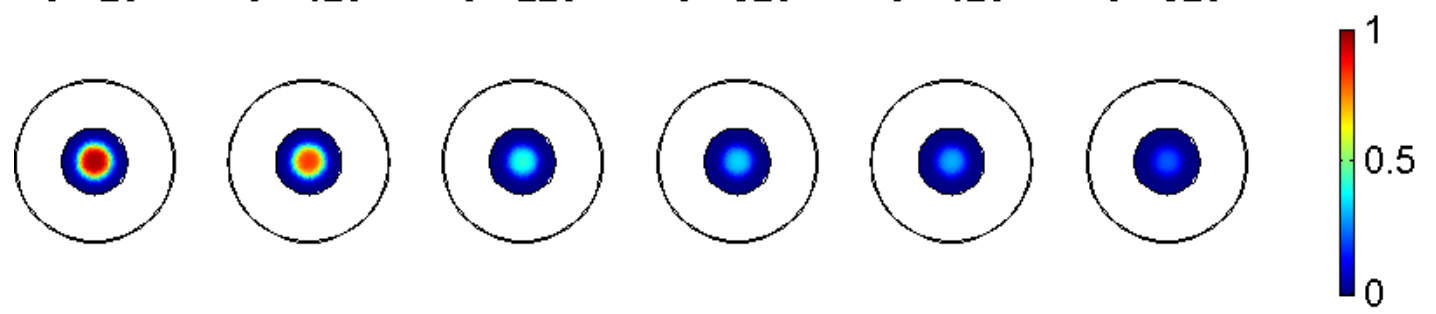

(a) $\mathrm{N}$
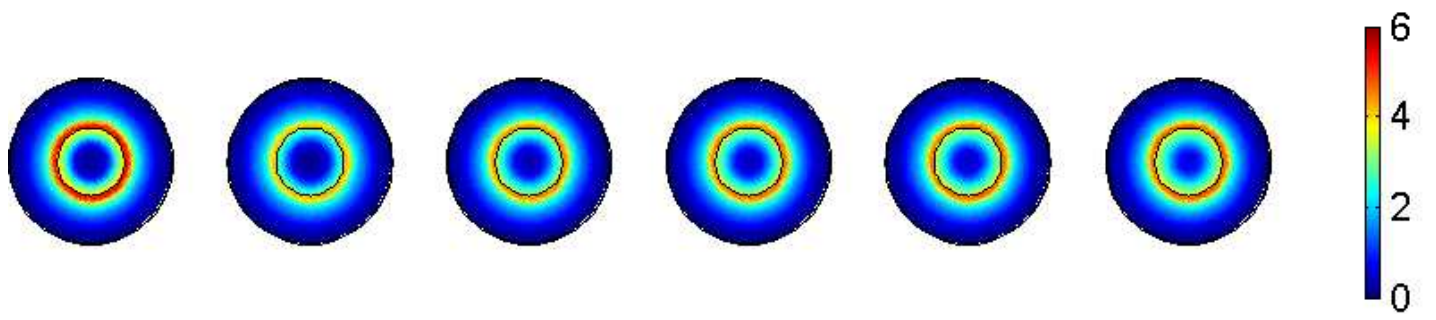

(b) ATMi
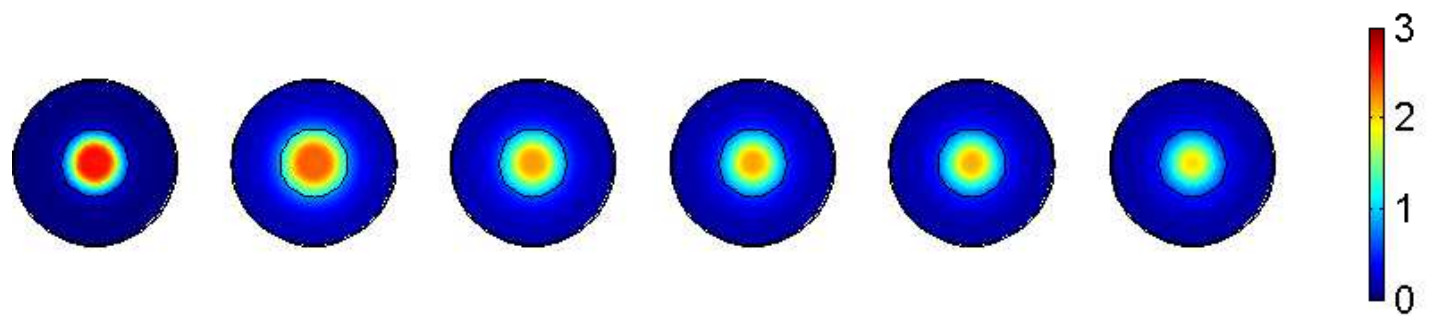

(c) ATMp
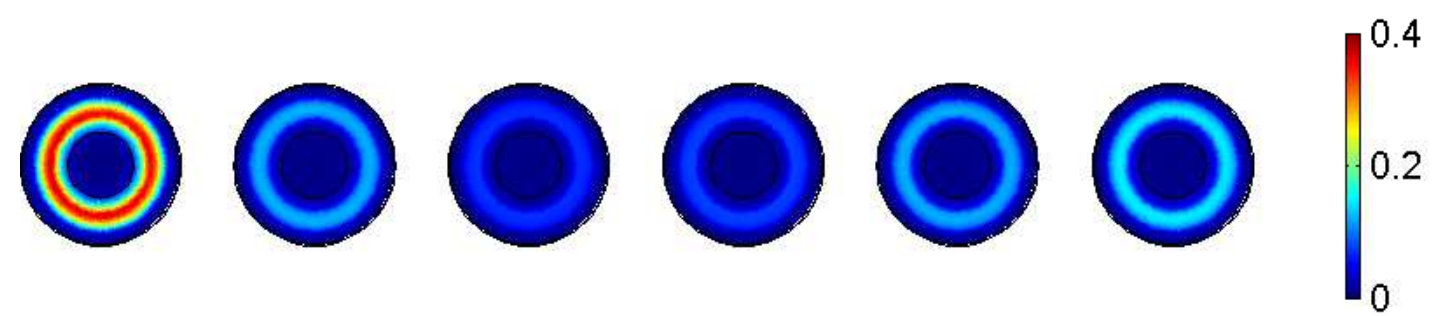

(d) $\mathrm{p} 53 \mathrm{i}$
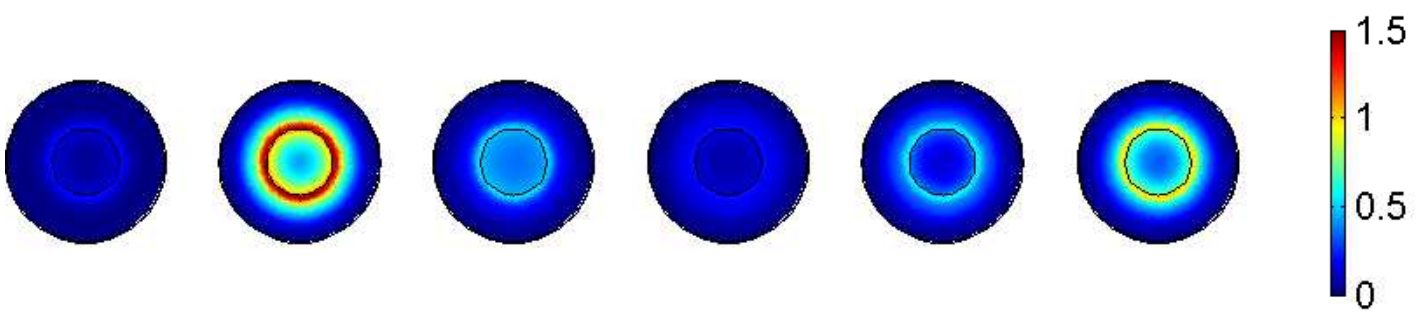

(e) p53p

Figure 5. Spatial profiles of various species in case 2 (see subsection 4.2 for a description). The local concentrations are shown in non-dimensional units, and the time is in dimensional units and shifted as in figure 4. 


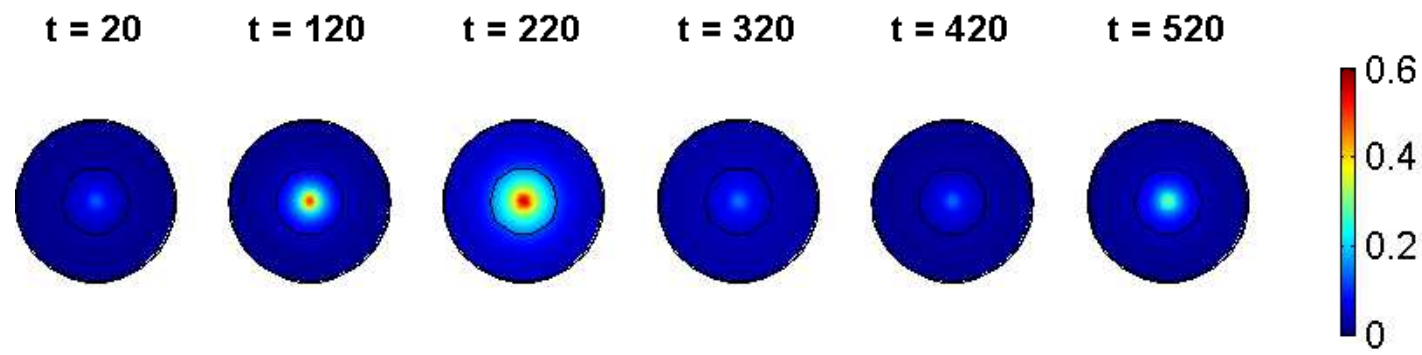

(a) Mdm2 transcript
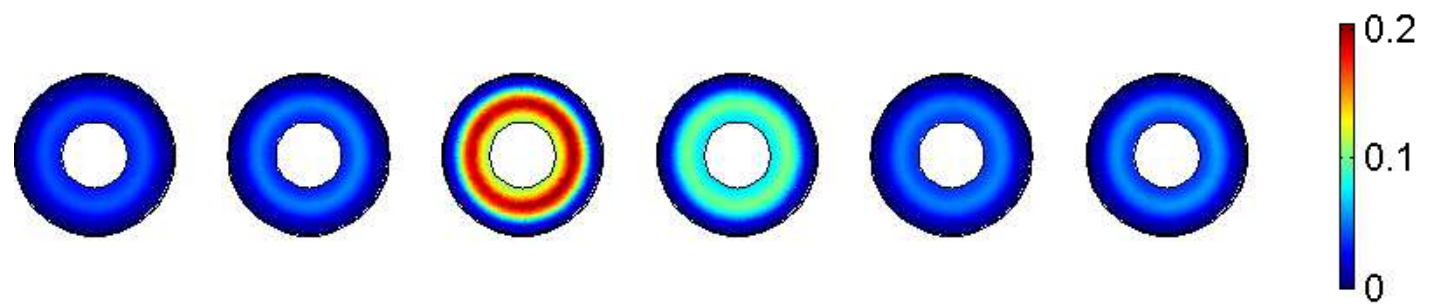

(b) $\operatorname{Mdm} 2 \mathrm{i}$
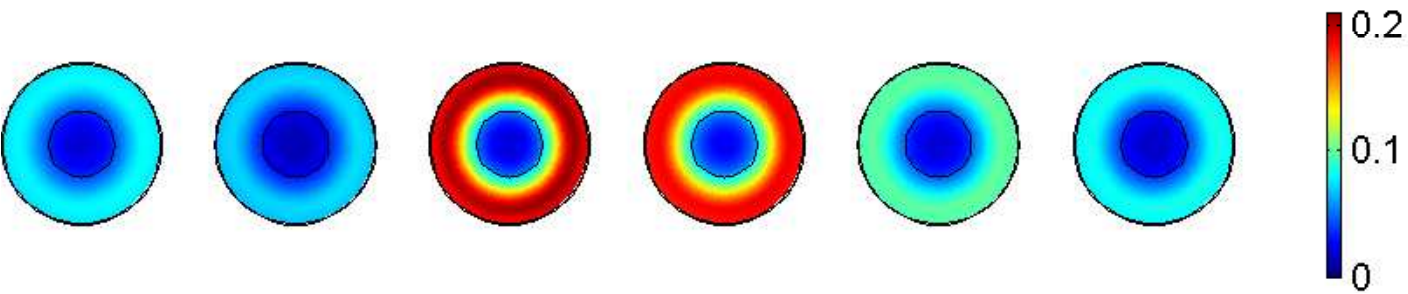

(c) $\operatorname{Mdm} 2 p$
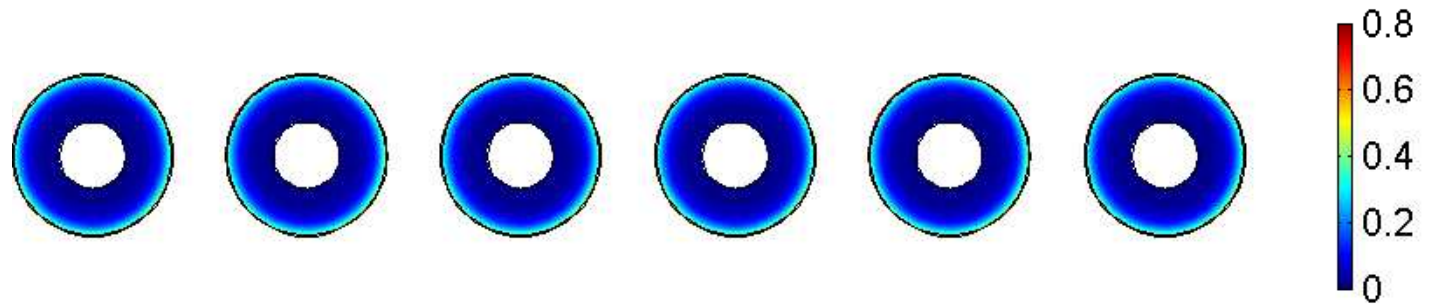

(d) Aktp

FiguRE 6. Spatial profiles of various species in case 2 (see subsection 4.2 for a description). The local concentrations are shown in non-dimensional units, and the time is in dimensional units and shifted as in figure 4.

and 2 are also radially symmetric about the cell centre), whereas our spatio-temporal results for cases 3 and 4 are spatially asymmetric (which reflects the spatially asymmetric modelling assumptions for cases 3 and 4). In particular, in cases 3 and 4, DNA damage occurs mostly on the left side of the nucleus, causing a higher rate of conversion of ATMi to ATMp on the left side of the nucleus. Higher levels of ATMp on the left side of the nucleus lead to greater conversion of p53i to p53p, and greater degradation of Mdm2i and Mdm2p, on the left side of the cell. Finally, the greater conversion of p53i to p53p on the left side of the cell leads to more rapid repair of the DNA damage that is furthest to the left in the nucleus, where it is closest to the nuclear membrane. 
In figure 7(b), we give a small demonstration of how the asymmetrical spatial distribution of p53p in case 4 compares with the spatial distribution of p53p in case 2 . The differences between all of the cases are most easily seen by viewing the various computational movie clips mentioned in section 6 .

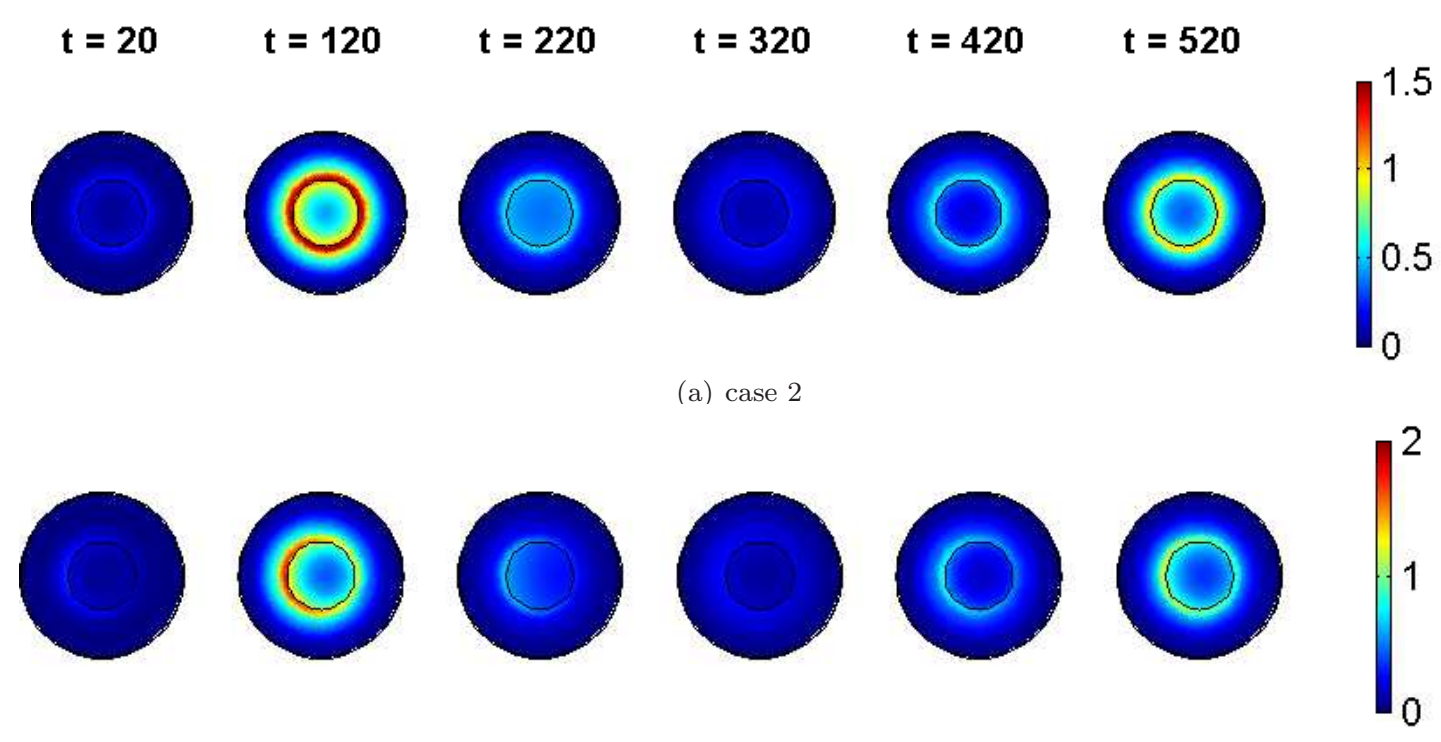

(b) case 4

Figure 7. Spatial profiles of p53p in: (a) case 2; (b) case 4. The local concentrations are shown in non-dimensional units, and the time is in dimensional units and shifted as in figure 4.

\subsection{Implications of our results}

Regarding the computational implications of our results, we see, from comparing our results for cases 3 and 4 with our results for cases 1 and 2, respectively, that a simple change to the spatial assumptions of our model may not significantly affect its temporal predictions at the same time that it does noticeably affect its spatio-temporal predictions. Clearly it would be impossible to gain this type of insight from a purely temporal model, such as a model composed of ordinary or delay differential equations. Thus, our results help to demonstrate the advantages of working with a spatio-temporal model.

Now we comment on the biological implications of our results. Our results from cases 2 and 4 (specifically, from figure 5(a) above and from figure 8.2(a) in the Supporting Information text file) show that DNA damage is repaired more quickly the closer it is to the nuclear membrane. In a real-world cell, if some genes are repaired more quickly than others, then this may influence cell behaviour, depending on which genes are affected. From cases 3 and 4 (for example, see figure 7(b) above), we know that levels of active p53 (p53p) can be higher on one side of the nucleus. The uneven spatial distribution of active p53 in the nucleus of a real-world cell could strongly influence the behaviour of the cell, because p53 has hundreds of target genes that are distributed on various chromosomes and that can be thought of as being in competition with each other to be activated by p53 [43]. Examples of p53-inducible genes in humans, and the chromosomes on which they are found, are as follows: Mdm2 gene, chromosome 12 [33]; PTEN gene, chromosome 10 [8]; PUMA gene, chromosome 19 [15]; BAX gene, chromosome 19 [1]; p21 gene, chromosome 6 [11]. Competition for expression amongst p53-inducible genes would be exacerbated if the total concentration of nuclear active p53 were to drop to a low level, which could happen if one of the gene copies for p53 were mutated. The fact that mutations in the p53 gene occur frequently in many 
common cancers is hardly likely to be a coincidence [43]. The spatial distribution of active p53 in the nucleus is significant to a cell not only because p53 is a transcription factor; there is evidence that p53 can be directly involved in DNA repair as a protein and not as a transcription factor $[26,35]$.

\section{Discussion}

In mammalian cells, the p53 pathway regulates the response to a variety of stresses, including oncogene activation, heat and cold shock, and DNA damage. Here we have explored a mathematical model of this pathway, composed of a system of partial different equations. In our model, the p53 pathway is activated by a DNA-compromising event of short duration. As is typical for mathematical models of the p53 pathway, our model contains a negative feedback loop representing interactions between the p53 and Mdm2 proteins. A novel feature of our model is that we combine a spatio-temporal approach with the appearance and repair of DNA damage.

Through numerical simulations using the software package COMSOL, we have investigated four cases. In case 1, we made spatial assumptions that were radially symmetric about the cell centre. We also ignored the possibility of DNA repair, allowing us to model the scenario in which the cell had a very inefficient DNA repair mechanism. We found that spatio-temporal oscillations in p53 and Mdm2 may occur, consistent with experimental results $[4,18]$. Our assumptions in case 2 were identical to those in case 1, except that active p53 was allowed to directly repair DNA damage. That active p53 may be directly involved in DNA repair is supported by experimental evidence $[26,35]$. We found in case 2 that oscillations in p53 and Mdm2 can still occur, but their amplitude damps down quickly as the DNA damage is repaired. Our modelling assumptions for cases 3 and 4 were the same as for, respectively, cases 1 and 2, with one exception: the centre of the region of DNA damage was located at the centre of the nucleus in cases 1 and 2, but was located to the left of the centre of the nucleus by a distance of one-tenth of the diameter of the nucleus in cases 3 and 4 . Although this simple difference in the location of the DNA damage did not significantly affect the temporal predictions of the model, it did cause some noticeable differences to its spatio-temporal predictions. In particular, in cases 3 and 4 , levels of active p53 were higher on the left side of the nucleus, whilst in cases 1 and 2, they were the same on both sides of the nucleus. These findings prompted us to recognise that the uneven spatial distribution of active p53 in the nucleus of a real-world cell could strongly influence the behaviour of the cell, because there are hundreds of p53-inducible genes which can be thought of as being in competition with each other to be activated by $\mathrm{p} 53$.

Our model contains only one p53-inducible gene, namely the Mdm2 gene. Therefore, one extension of our work would be to include many p53-inducible genes in our model. This extension would allow us to study how the spatial distribution of nuclear active p53 influences which genes are activated by it. Examples of genes that could be included in such an extension are the genes for PUMA and BAX. An advantage of introducing PUMA and BAX into our model would be that this would enable us to incorporate a mechanism for apoptosis into it, since PUMA and BAX are pro-apototic proteins [30].

\section{Supporting Information}

Text file "SI_text". This file includes the statement of the full system of equations for our model, details about its non-dimensionalisation, details about simulating the non-dimensionalised model, and calculations of parameter values in the dimensional model. Simulation results are also included.

Computational movie clips. Movie clips showing local concentration results. Concentrations and times are shown in non-dimensionalised units, with the DNA-compromising event lasting from time 600 to time 620 .

- Movie clips A1, A2, and A3 show, respectively, total p53 (p53i + p53p), p53p, and Mdm2p for the case (case 1) considered in subsection 4.1. 
- Movie clips B1, B2, B3, and B4 show, respectively, DNA damage N, total p53 (p53i + p53p), p53p, and $\mathrm{Mdm} 2 \mathrm{p}$ for the case (case 2) considered in subsection 4.2.

- Movie clips C1, C2, and C3 show, respectively, total p53 (p53i + p53p), p53p, and Mdm2p for case 3, which is mentioned in subsection 4.3 .

- Movie clips D1, D2, D3, and D4 show, respectively, DNA damage N, total p53 (p53i + p53p), p53p, and Mdm2p for case 4, which is mentioned in subsection 4.3.

Movie clips A1, A2, B2, B3, C1, C2, D2, and D3 compare favourably with experimental movie clips of p53 oscillations made using fluorescent fusion proteins in MCF-7 cells (see [25] and the supporting information files in $[13,17,18])$.

Acknowledgements. This work was supported by the European Research Council, specifically the Advanced Investigator Grant 227619-M5CGS with title "From Mutations to Metastases: Multiscale Mathematical Modelling of Cancer Growth and Spread".

\section{References}

[1] S.S. Apte, M.G. Mattei, B.R. Olsen. Mapping of the human BAX gene to chromosome 19q13.3-q13.4 and isolation of a novel alternatively spliced transcript, BAX delta. Genomics, 26 (1995), 592-594.

[2] C.J. Bakkenist, M.B. Kastan. DNA damage activates ATM through intermolecular autophosphorylation and dimer dissociation. Nature, 421 (2003), 499-506.

[3] E. Batchelor, C.S. Mock, I. Bhan, A. Loewer, G. Lahav. Recurrent initiation: A mechanism for triggering p53 pulses in response to DNA damage. Molecular Cell, 30 (2008), 277-289.

[4] E. Batchelor, A. Loewer, G. Lahav. The ups and downs of p53: understanding protein dynamics in single cells. Nature Reviews Cancer, 9 (2009), 371-377.

[5] C. Blattner, T. Hay, D.W. Meek, D.P. Lane. Hypophosphorylation of Mdm2 augments p53 stability. Molecular and Cellular Biology, 22 (2002), 6170-6182.

[6] A.M. Bode, Z. Dong. Post-translational modifications of p53 in tumorigenesis. Nat. Rev. Cancer, 4 (2004), $793-805$.

[7] A. Cangiani, R. Natalini. A spatial model of cellular molecular trafficking including active transport along microtubules. J. Theor. Biol., 267 (2010), 614-625.

[8] A. Carracedo, A. Alimonti, P.P. Pandolfi. PTEN level in tumor suppression: how much is too little? Cancer Research, 71 (2011), 629-633.

[9] C.H. Chung, K. Ely, L. McGavran, M. Varella-Garcia, J. Parker, N. Parker, C. Jarrett, J. Carter, B.A. Murphy, J. Netterville, B.B. Burkey, R. Sinard, A. Cmelak, S. Levy, W.G. Yarbrough, R.J. Slebos, F.R. Hirsch. Increased epidermal growth factor receptor gene copy number is associated with poor prognosis in head and neck squamous cell carcinomas. J. Clin. Oncol., 24 (2006), 4170-4176.

[10] A. Ciliberto, B. Novak, J. Tyson. Steady states and oscillations in the p53/Mdm2 network. Cell Cycle, 4:3 (2005), 488-493.

[11] S.E. Coupland, N. Bechrakis, A. Schuler, I. Anagnostopoulos, M. Hummel, N. Bornfeld, H. Stein. Expression patterns of cyclin D1 and related proteins regulating G1-S phase transition in uveal melanoma and retinoblastoma. Br. J. Ophthalmol., 82 (1998), 961-970.

[12] L. Dimitrio, J. Clairambault, R. Natalini. A spatial physiological model for p53 intracellular dynamics. J. Theor. Biol., 316 (2012), 9-24.

[13] N. Geva-Zatorsky, N. Rosenfeld, S. Itzkovitz, R. Milo, A. Sigal, E. Dekel, T. Yarnitzky, Y. Liron, P. Polak, G. Lahav, U. Alon. Oscillations and variability in the p53 system. Mol. Sys. Biol., 2 (2006), 0033.

[14] P. Giannakakou, D.L. Sackett, Y. Ward, K.R. Webster, M.V. Blagosklonny, T. Fojo. p53 is associated with cellular microtubules and is transported to the nucleus by dynein. Nat. Cell Biol., 2 (2000), 709-717.

[15] P. Hikisz, Z.M. Kilianska. Puma, a critical mediator of cell death - one decade on from its discovery. Cell. Mol. Biol. Lett., 17 (2012), 646-669.

[16] K.K. Khanna, M.F. Lavin, S.P. Jackson, T.D. Mulhern. ATM, a central controller of cellular responses to DNA damage. Cell Death Diff., 8 (2001), 1052-1065.

[17] G. Lahav, N. Rosenfield, A. Sigal, N. Geva-Zatorsky, A.J. Levine, M.B. Elowitz, U. Alon. Dynamics of the p53-Mdm2 feedback loop in individual cells. Nat. Gen., 36 (2004), 147-150.

[18] A. Loewer, E. Batchelor, G. Gaglia, G. Lahav. Basal dynamics of p53 reveal transcriptionally attenuated pulses in cycling cells. Cell, 142 (2010), 89-100.

[19] L. Ma, J. Wagner, J.J. Rice, W. Hu, A.J. Levine, G.A. Stolovitzky. A plausible model for the digital response of $p 53$ to DNA damage. Proc. Natl. Acad. Sci. USA, 102 (2005), 14266-14271.

[20] J.J. Manfredi. The Mdm2-p53 relationship evolves: Mdm2 swings both ways as an oncogene and a tumor suppressor. Genes \& Development, 24 (2010), 1580-1589.

[21] J.C. Marine. p53 stabilization: the importance of nuclear import. Cell Death Diff., 17 (2010), $191-192$. 
[22] L.D. Mayo, D.B. Donner. A phosphatidylinositol 3-kinase/Akt pathway promotes translocation of Mdm2 from the cytoplasm to the nucleus. Proc. Natl. Acad. Sci. USA, 98 (2001), 11598-11603.

[23] G.I. Mihalas, M. Neamtu, D. Opris, R.F. Horhat. A dynamic P53-MDM2 model with time delay. Chaos, Solitons and Fractals, 30 (2006), 936-945.

[24] N. Monk. Oscillatory expression of Hes1, p53, and NF- $\kappa B$ driven by transcriptional time delays. Curr. Biol., 13 (2003), 1409-1413.

[25] C.C. Morton. Cancer sentry flashes two-tiered warning, http://hms.harvard.edu/news/cancer-sentry-flashes-twotiered-warning-9-3-10, 2010.

[26] H. Offer, M. Milyavsky, N. Erez, D. Matas, I. Zurer, C.C. Harris, V. Rotter. Structural and functional involvement of p53 in BER in vitro and in vivo. Oncogene, 20 (2001), 581-589.

[27] Y. Ogawara, S. Kishishita, T. Obata, Y. Isazawa, T. Suzuki, K. Tanaka, N. Masuyama, Y. Gotoh. Akt enhances Mdm2-mediated ubiquitination and degradation of p53. J. Biol. Chem., 277 (2002), 21843-21850.

[28] D.A. Ouattara, W. Abou-Jaoude, M. Kaufman. From structure to dynamics: Frequency tuning in the p53-Mdm2 network. II Differential and stochastic approaches. J. Theor. Biol., 264 (2010), 1177-1189.

[29] C.J. Proctor, D.A. Gray. Explaining oscillations and variability in the p53-Mdm2 system. BMC Systems Biology, 2 (2008), 1-20.

[30] T. Pu, X.P. Zhang, F. Liu, W. Wang. Coordination of the nuclear and cytoplasmic activities of p53 in response to DNA damage. Biophysical Journal, 99 (2010), 1696-1705.

[31] K. Puszynski, B. Hat, T. Lipniacki. Oscillations and bistability in the stochastic model of p53 regulation. J. Theor. Biol., 254 (2008), 452-465.

[32] K. Puszynski, R. Bertolusso, T. Lipniacki. Crosstalk between p53 and NF- $\kappa$ B systems: pro- and anti-apoptotic functions of $N F-\kappa B$. IET Sys. Biol., 3 (2009), 356-367.

[33] P. Ragazzini, G. Gamberi, M.S. Benassi, C. Orlando, R. Sestini, C. Ferrari, L. Molendini, M.R. Sollazzo, M. Merli, G. Magagnoli, F. Bertoni, T. Bohling, M. Pazzagli, P. Picci. Analysis of SAS gene and CDK4 and MDM2 proteins in low-grade osteosarcoma. Cancer Detection and Prevention, 23 (1999), 129-136.

[34] D.R. Schrider, M.W. Hahn. Gene copy-number polymorphism in nature. Proc. Roy. Soc. B, 277 (2010), 3213-3221.

[35] M.L. Smith, Y.R. Seo. p53 regulation of DNA excision repair pathways. Mutagenesis, 17 (2002), $149-156$.

[36] V. Stambolic, D. MacPherson, D. Sas, Y. Lin, B. Snow, Y. Jang, S. Benchimol, T.W. Mak. Regulation of PTEN transcription by p53. Mol. Cell, 8 (2001), 317-325.

[37] A.H. Stegh. Targeting the p53 signaling pathway in cancer therapy - the promises, challenges and perils. Expert Opinion on Therapeutic Targets, 16 (2012), 67-83.

[38] J.M. Stommel, G.M. Wahl. A new twist in the feedback loop: stress-activated MDM2 destabilisation is required for p53 activation. Cell Cycle, 4 (2005), 411-417.

[39] M. Sturrock, A.J. Terry, D.P. Xirodimas, A.M. Thompson, M.A.J. Chaplain. Spatio-temporal modelling of the Hes1 and p53-Mdm2 intracellular signalling pathways. J. Theor. Biol., 273 (2011), 15-31.

[40] M. Sturrock, A.J. Terry, D.P. Xirodimas, A.M. Thompson, M.A.J. Chaplain. Influence of the nuclear membrane, active transport, and cell shape on the Hes1 and p53-Mdm2 pathways: insights from spatio-temporal modelling. B. Math. Biol., 74 (2012), 1531-1579.

[41] A.J. Terry, M. Sturrock, J.K. Dale, M. Maroto, M.A.J. Chaplain. A spatio-temporal model of Notch signalling in the zebrafish segmentation clock: conditions for synchronised oscillatory dynamics. PLoS ONE, 6 (2011), e16980.

[42] A.J. Terry, M.A.J. Chaplain. Spatio-temporal modelling of the NF- $\kappa B$ signalling pathway: The roles of diffusion, active transport, and cell geometry. J. Theor. Biol., 290 (2011), 7-26.

[43] R. Weinberg. The Biology of Cancer. Garland Science: Taylor \& Francis Group, 2007.

[44] D.P. Xirodimas, C.W. Stephen, D.P. Lane. Cocompartmentalization of $p 53$ and Mdm2 is a major determinant for Mdm2-mediated degradation of p53. Experimental Cell Research, 270 (2001), 66-77.

[45] T. Zhang, P. Brazhnik, J.J. Tyson. Exploring mechanisms of the DNA-damage response: p53 pulses and their possible relevance to apoptosis. Cell Cycle, 6 (2007), 85-94. 